\title{
Understanding the mathematics of hearing using electronic circuits
}

\author{
T. J. Hamilton ${ }^{1} \quad$ J. Tapson ${ }^{2} \quad$ M. Rapson ${ }^{3} \quad$ C. Jin ${ }^{4}$ \\ A. van Schaik ${ }^{5}$
}

(Received 25 January 2010; revised 22 May 2010)

\begin{abstract}
The human cochlea is a fascinating transduction organ that illustrates the ingenious way in which engineering problems are solved in nature. A healthy cochlea has a dynamic range in the order of $120 \mathrm{~dB}$; that is, the difference between the roar of the engines of a Boeing 747 and the faintest whisper. We discuss the recent assertion that the cochlea is governed by the dynamics of a Hopf bifurcation. In our cochlea model we discretise the basilar membrane into resonant sections with logarithmically decreasing characteristic frequencies. We show that the observed active behaviour of the cochlea can be modelled as a change in the quality factor of the individual resonant sections in a discretised model, and that this has dynamics which embody the Hopf bifurcation.
\end{abstract}

http://anziamj.austms.org.au/ojs/index.php/ANZIAMJ/article/view/2543 gives this article, (c) Austral. Mathematical Soc. 2010. Published June 17, 2010. ISSN 1446-8735. (Print two pages per sheet of paper.) 


\section{Contents}

1 Introduction

C301

2 Characteristics of the active cochlea and the Hopf bifurcation

C302

2.1 Active, nonlinear characteristics of the cochlea . . . . . . C302

2.2 The Hopf bifurcation . . . . . . . . . . . . . . . C305

3 The cochlea model

C306

4 Results

C310

5 Conclusions

C313

References

C313

\section{Introduction}

The cochlea is a small, bony organ in the inner ear which transduces mechanical energy into electrical signals. It has been the object of neuro-scientific research for almost 150 years. The interest is mainly due to its huge dynamic range $(\approx 120 \mathrm{~dB}[9])$ and ability to adapt to a wide variety of listening environments. The existence of an active cochlear amplifier is well accepted, and the active elements are generally agreed to be the outer hair cells (OHCs). In 2000, Eguíluz et al. [2] proposed that there was strong evidence that the cochlear amplifier has the dynamical characteristics of a Hopf bifurcation. Camalet et al. [1] made an almost simultaneous conjecture, specifying that the Hopf bifurcation was a result of self-tuned critical oscillations of the hair cells. Kern and Stoop [6, 7] subsequently developed a coupled structure that suggests that sections of the cochlea cannot be considered in isolation, as their dynamics affect the propagation of sound through the cochlea as a 
whole. Magnasco [8] developed an alternative coupled model which shows somewhat different results.

We present a two dimensional (2D) model of the cochlea which includes supercritically stable amplification (a Hopf bifurcation) in a physiologically realistic way. We show that the nonlinearities predicted in other models $[1$, $2,6,7,8]$ are present in this physical system, and that successful operation in the supercritical regime is feasible, thereby confirming that a cochlea with physiologically plausible coupling can operate in this fashion.

\section{Characteristics of the active cochlea and the Hopf bifurcation}

\subsection{Active, nonlinear characteristics of the cochlea}

In our discussions on the biological cochlea we use the terminology active to emphasise the fact that the cochlea is a dynamic organ, expending energy to enhance the received sound. Figure 1 shows the simplified, uncoiled cochlea. Here we see both the oval window, which is connected to the stapes, and the round window, which is a membrane that allows the pressure within the cochlea duct to be equalised. The fluid within the cochlea is assumed to be incompressible. When the oval window moves, the basilar membrane (BM) is deflected and the round window moves in the opposite direction to the initial movement in the oval window.

The BM changes in width and elasticity from narrow and stiff at the base to wide and flexible at the apex. These changes in the physical characteristics of the BM assist to separate an input signal into its frequency components. At the base of the cochlea the physical characteristics of the BM are such that it responds better (that is, greater movement is produced) to high frequency stimuli whereas the apex responds better to low frequency stimuli. The 


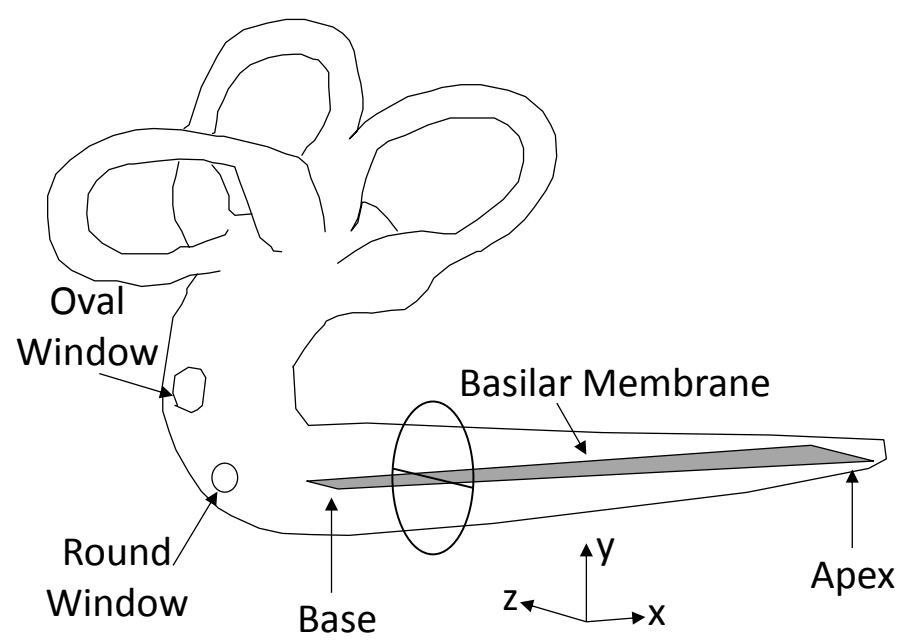

Figure 1: The uncoiled cochlea.

characteristic frequency at a particular place along the BM is said to be the frequency that produces the greatest deflection at that place.

Figure 2 shows the nonlinear and active characteristics measured from the chinchilla cochlea. Figure 2(a) plots frequency versus BM velocity (a measure of frequency response at a particular place in the cochlea). For large input signals the frequency response is highly damped. For small input signals the frequency response is more highly tuned.

Figure 2(b) shows the gain for each input intensity. This figure illustrates the nonlinear nature of the active gain. Here we see that for small input intensities the gain is much larger than for large input intensities. This phenomenon is called large-signal compression. 

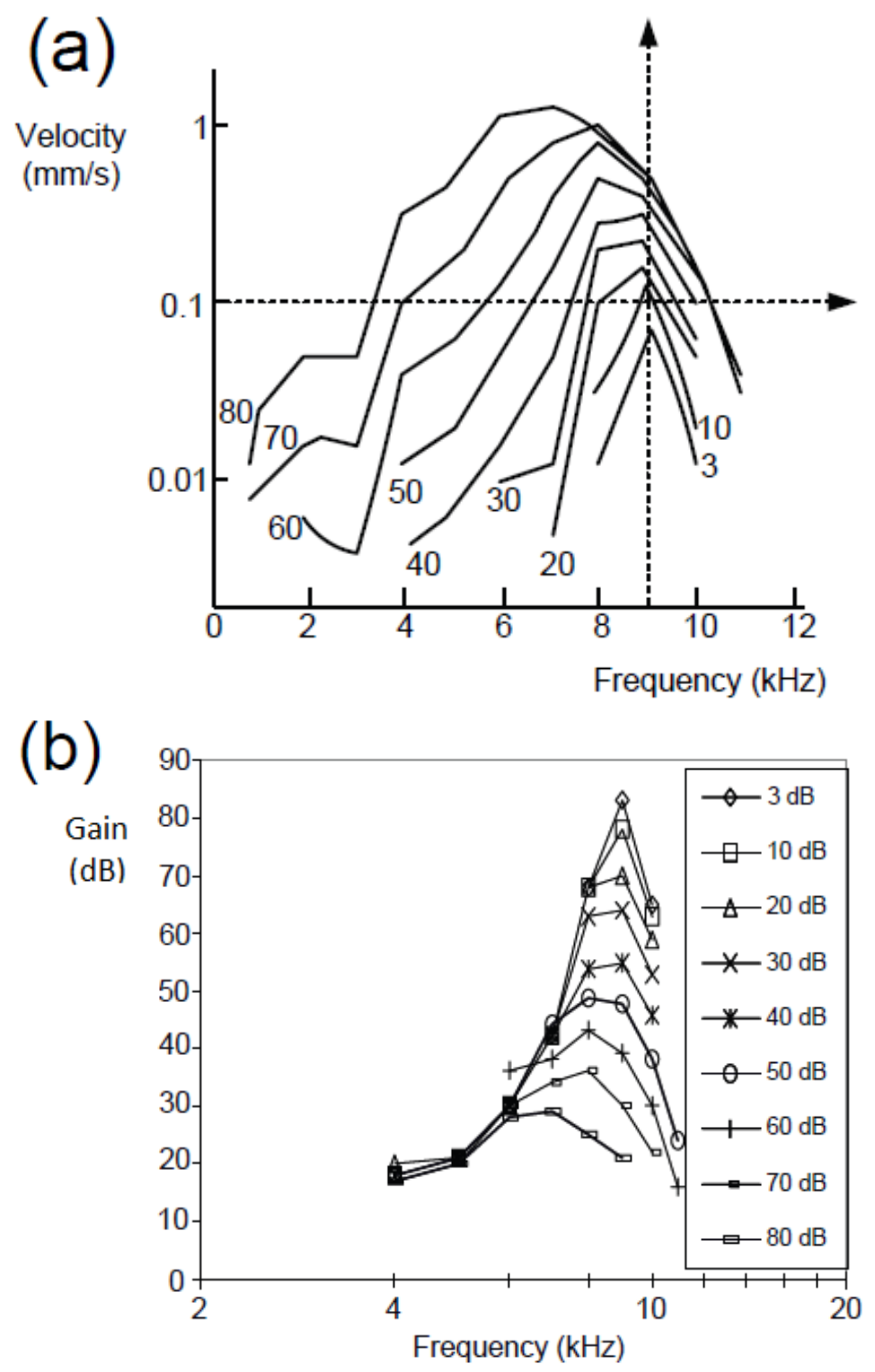

FiguRE 2: Frequency response at a particular place along the basilar membrane in a chinchilla cochlea where the characteristic frequency is $9 \mathrm{kHz}$ [10]. 


\subsection{The Hopf bifurcation}

A Hopf bifurcation is a critical point in a nonlinear system where there is a transition between a stable equilibrium point and a limit cycle. A feature of the (supercritical) Hopf bifurcation is that there is a smooth transition between this stable equilibrium and the limit cycle and back again. This is an important feature for a biological system where there is sure to be mismatch and noise that may otherwise (for example, if the Hopf bifurcation is subcritical) push it into an unstable region without the ability to re-establish its correct operating point. A differential equation that embodies the Hopf bifurcation for a single variable is [2]

$$
\frac{d z}{d t}=\left(\mu+i \omega_{0}\right) z-z^{3}+F e^{i \omega t},
$$

where $z(t)$ is the output signal and a complex variable of time of the form $z(t)=x(t)+i y(t), \mu$ is the control parameter (set-point), $\omega_{0}$ is the resonant frequency and $\mathrm{Fe}^{\mathrm{i \omega t}}$ is a forcing function provided by some external force. Note that the two dimensions (variables) used to describe the normal form of the Hopf bifurcation are encompassed by the real and imaginary parts of $z$.

In (1) when the forcing function is excluded the control parameter $\mu$ is varied to obtain stable $(\mu<0)$, critical $(\mu=0)$ and unstable (limit cycle) $(\mu>0)$ solutions. When operating at the bifurcation point $(\mu=0)$ the system is said to be supercritically stable.

When the forcing function is included, the dynamics of the system become significantly more complicated. Eguíluz et al. [2] discusses the full range of entrainment behaviour that can occur. When the system is supercritically stable, and it is driven at $\omega_{0}$, the response is nonlinear with a high gain for small forcings and smaller gains for larger forcings. If it is driven at $\omega=\omega_{0}+\Delta \omega, \Delta \omega \neq 0$, then the response is approximately linear for sufficiently small input.

Therefore the cubic term in (1) introduces a compressive nonlinearity at resonance. This compressive nonlinearity contributes to the damped response for 
large inputs and highly tuned response for small inputs. Hence, equation (1) possesses important dynamics observed in the mammalian cochlea.

\section{The cochlea model}

Our active cochlea model is based on the passive models developed by Fragniere [3] and van Schaik et al. [13]. It consists of a resistive network simulating a fluid region and resonators modelling the basilar membrane behaviour. The fluid is assumed to be inviscid, incompressible and irrotational. Under these conditions, Fragniere [3] showed that it can be modelled by a resistive network that is equivalent to a finite difference approximation to the Laplace equation

$$
\frac{\partial^{2} p(x, y)}{\partial x^{2}}+\frac{\partial^{2} p(x, y)}{\partial y^{2}}=0
$$

where $p(x, y)$ is the fluid pressure at the location $(x, y)$, represented as a voltage in the network. Newton's second law provides a relationship between pressure and the $x$ and $y$ acceleration in the fluid

$$
\frac{\partial p(x, y)}{\partial x}=\rho(x, y) a_{x}(x, y), \quad \frac{\partial p(x, y)}{\partial y}=\rho(x, y) a_{y}(x, y) .
$$

Either the pressure or its normal gradient (proportional to the acceleration) must be specified at each location on the boundary of the fluid region. The BM boundary condition is described below and the oval window, which serves as the input to the cochlea, is represented by the $y$-axis (that is $x=0$ ) boundary. The helicotrema is modelled by a pressure of zero at the apex and the remaining boundaries represent the wall of the cochlea where acceleration is assumed to be zero.

Our 2D cochlea model is illustrated in Figure 3. The resonators are shown along the BM which is represented by the $x$-axis (that is $y=0$ ). The characteristic frequency of the resonators logarithmically decreases from base to 


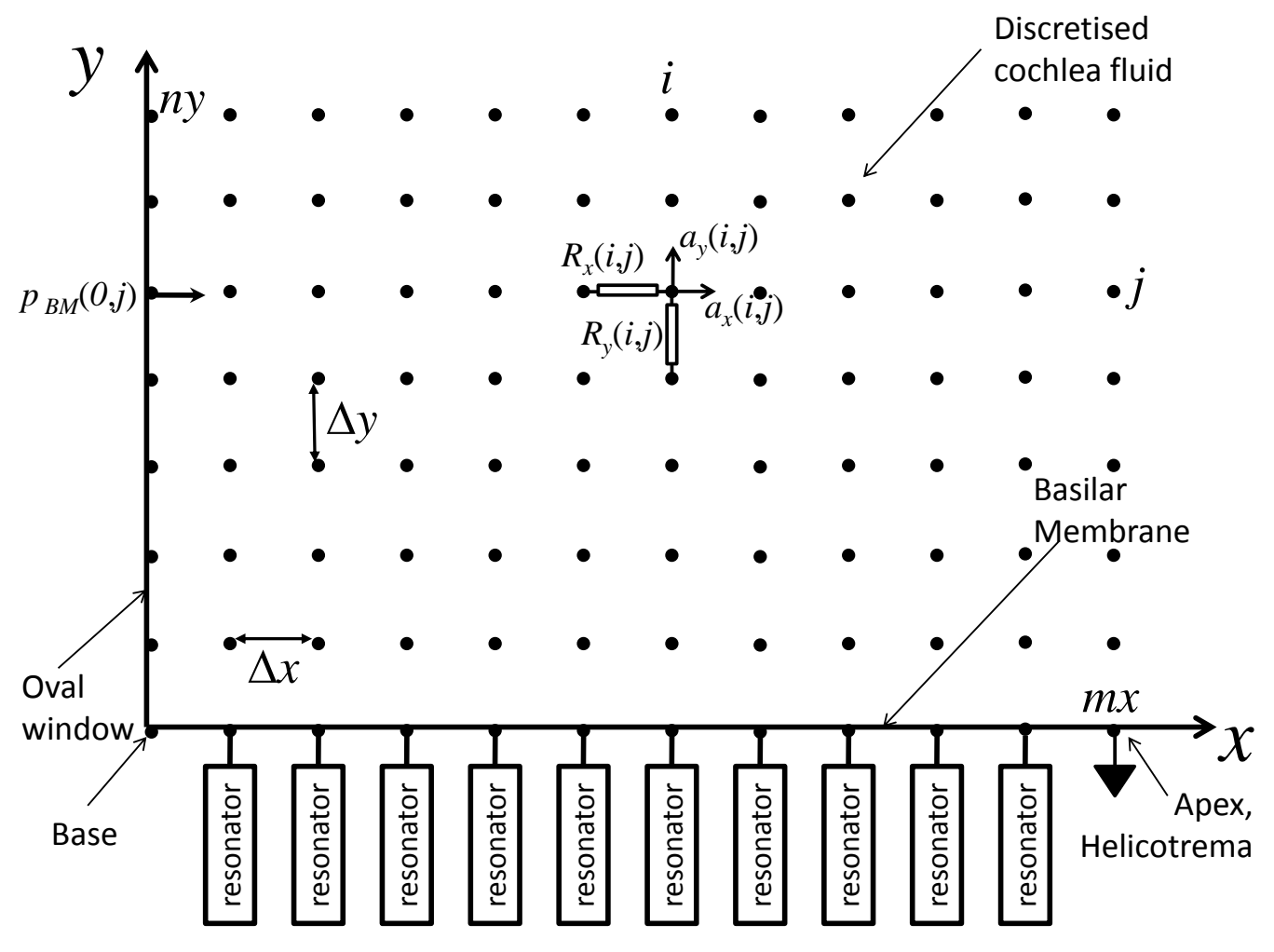

Figure 3: The 2D cochlea model.

apex as in the real cochlea. The resonators are coupled together through the cochlea fluid which is discretised into $m \times n$ sections. Resistor values, $R_{x}(i, j)$ and $R_{y}(i, j)$, depend on $\rho$ and the size of each section.

A simplifed equation describing the motion of BM at a point $(x, 0)$ on the boundary of the fluid is

$$
\Delta \mathrm{p}_{\mathrm{BM}}(\mathrm{x}) w(x) \mathrm{d} x=\mathrm{a}_{\mathrm{BM}}(\mathrm{x})\left\{\mathrm{m}(\mathrm{x}) \mathrm{d} x+\frac{\mathrm{h}(\mathrm{x})}{\mathrm{s}} \mathrm{d} x+\frac{\mathrm{k}(\mathrm{x})}{\mathrm{s}^{2}} \mathrm{~d} x\right\},
$$

where $\Delta p_{\mathrm{BM}}(x)=2 p(x, 0)$ is the pressure difference across the BM. The 
factor of two accounts for the fact that only one scalar is modelled. $w(x)$ is the width of the BM, $a_{B M}(x)=-a(x, 0)$ is the acceleration of the BM, $m(x)$ is the mass of the BM, $h(x)$ is the damping associated with the BM, and $k(x)$ is the stiffness of the BM. In the passive model the width $w$, mass $\mathrm{m}$, and damping $h$, of the BM are assumed to be constant for the entire length of the BM. The stiffness term, $k$, is modelled by the logarithmically decreasing characteristic frequency along the length of the BM.

Equation (4) can be discretised into a set of individual resonators at given locations $i$ along the BM. The relationship between pressure and acceleration for a single resonator is

$$
a_{B M i}=\frac{s^{2}}{s^{2} m_{i}+s h_{i}+k_{i}} \Delta p_{B M i},
$$

where $s$ is a complex variable in the Laplace domain. Here we assume that the width, $w(x)$, is constant in a discrete segment, $\Delta x$, of the BM. Thus, for simplicity both of these terms are omitted.

The sensing cells in the cochlea, the inner hair cells (IHCs), transduce the BM velocity into a neural signal. BM velocity is thus taken as the output for each resonator. Integrating (5) we obtain

$$
\frac{\mathrm{a}_{\mathrm{BMi}}}{\mathrm{s}}=v_{\mathrm{BMi}}=\frac{\mathrm{s}}{\mathrm{s}^{2} \mathrm{~m}_{\mathrm{i}}+s \mathrm{~h}_{\mathrm{i}}+\mathrm{k}_{\mathrm{i}}} \Delta \mathrm{p}_{\mathrm{BMi}},
$$

where $v_{\mathrm{BMi}}$ is the velocity at a particular place, $i$, along the BM. Equation (6) is proportional to the typical band-pass filter response given in (7), where $\Gamma$ is the filter output (analogous to BM velocity, $\mathrm{a}_{\mathrm{BMi}}$ ), $\theta$ is the filter input (analogous to the pressure change across the BM, $\left.\Delta \mathrm{p}_{\mathrm{BMi}}\right)$, $\tau$ is the time constant of the filter and $\mathrm{Q}$ is the quality factor

$$
\Gamma=\left(\frac{s \tau}{s^{2} \tau^{2}+s \tau / Q+1}\right) \theta .
$$

By observing the behaviour of the BM (see Figure 2) we see that the tuning of the response of the BM, that is the quality factor $\mathrm{Q}$ of the $\mathrm{BM}$ at a particular 


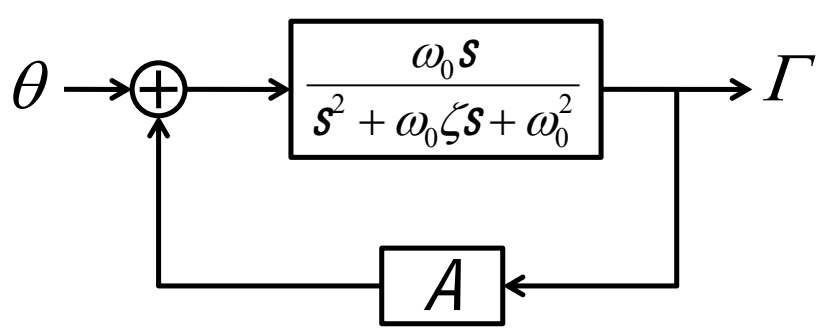

FigURE 4: BM resonant system with positive feedback.

place, is higher for small inputs than for large inputs. This observation is incorporated into our cochlea model by varying the $\mathrm{Q}$ of each resonator based on the intensity of the input signal. Looking at (6) and (7), Q is equivalent to the inverse of the damping term $h$. Thus, in the $2 \mathrm{D}$ active cochlea model we no longer consider the effective damping to be constant but rather dependent on BM velocity.

The adaptation of the $\mathrm{Q}$ for each resonator based on its input signal is achieved via a local feedback loop. Figure 4 illustrates the feedback loop for the BM resonant system. Here, $A$ is the feedback gain term, $\omega_{0}$ is the resonant frequency, equivalent to the inverse of the time constant $\tau$, and

$$
\zeta=\frac{1+\mathrm{AQ}}{\mathrm{Q}} .
$$

This resonant system can be described by

$$
\Gamma=\left(\frac{\omega_{0} s}{s^{2}+s \omega_{0} \zeta+\omega_{0}^{2}}\right)(\theta+A \Gamma),
$$

which is equivalent to (7). From (8) and (9) changing the feedback term $A$ is equivalent to changing the $\mathrm{Q}$ of the resonant system.

A feedback loop that adds the weighted energy of its output signal to its input, such as that shown in Figure 4, possesses the dynamical properties of a Hopf bifurcation [12]. This can be shown by rewriting (9) in differential 
form to obtain

$$
\theta+A \Gamma=\frac{1}{\omega_{0}} \frac{d \Gamma}{d t}+\zeta \Gamma+\int \omega_{0} \Gamma d t
$$

Select

$$
A=g\left(\mu-|\Gamma|^{2}\right)
$$

where $g$ is a constant gain factor and $\mu$ is the system set-point, we rewrite (10) as

$$
\frac{1}{\omega_{0}} \frac{d \Gamma}{d t}+(\zeta-g \mu) \Gamma+\int \omega_{0} \Gamma d t+g \Gamma|\Gamma|^{2}-\theta=0,
$$

so that it is in the form of a first order system that displays the dynamical properties of the Hopf bifurcation [11]. In (12) we are on the critical point, and hence, supercritically stable when $\zeta=g \mu$. Therefore, using this model we are able to tune the resonators to stable, critical and unstable operating points simply by varying $\mu$.

\section{Results}

The 2D cochlea model outlined in the previous section was implemented in several analogue VLSI hardware models. The hardware models included between 12 and 83 resonant sections. Further details of the hardware implementation are recorded elsewhere $[4,5]$. Note that in the figures below "dBV" refers to decibels relative to one Volt.

Figure 5 shows the frequency response (a) and the transient response (b) of the single resonator described by (9) and (12). Here we see the change in gain and selectivity (Q) as the input signal intensity varies. In Figure 5(b) the $\mu$ is set very close to the Hopf bifurcation and as such the gain between the input and the output is very large.

Figure 6 shows the frequency response (a) and the gain (b) of a single resonator at a particular place when the entire cochlea model is tested. Here we 

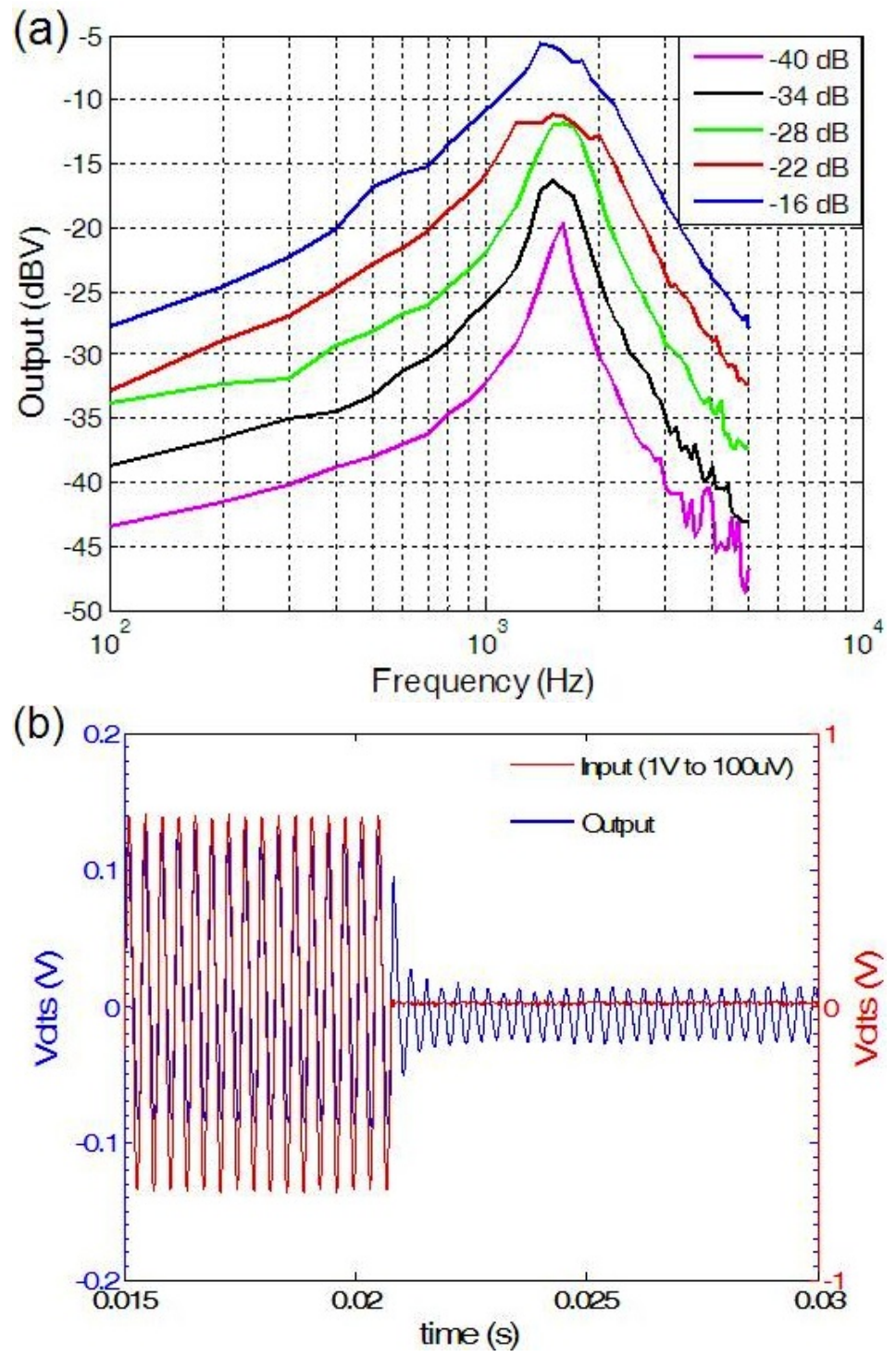

FiguRE 5: Frequency response (a) and transient response (b) of a single resonator when the input signal intensity is varied. 
(a)

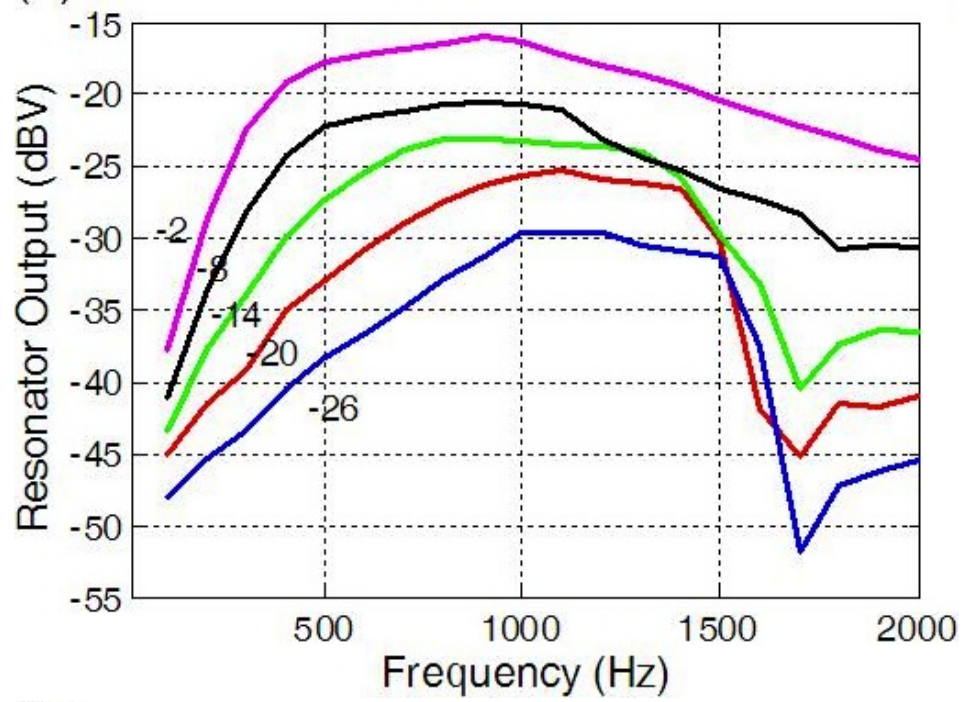

(b)

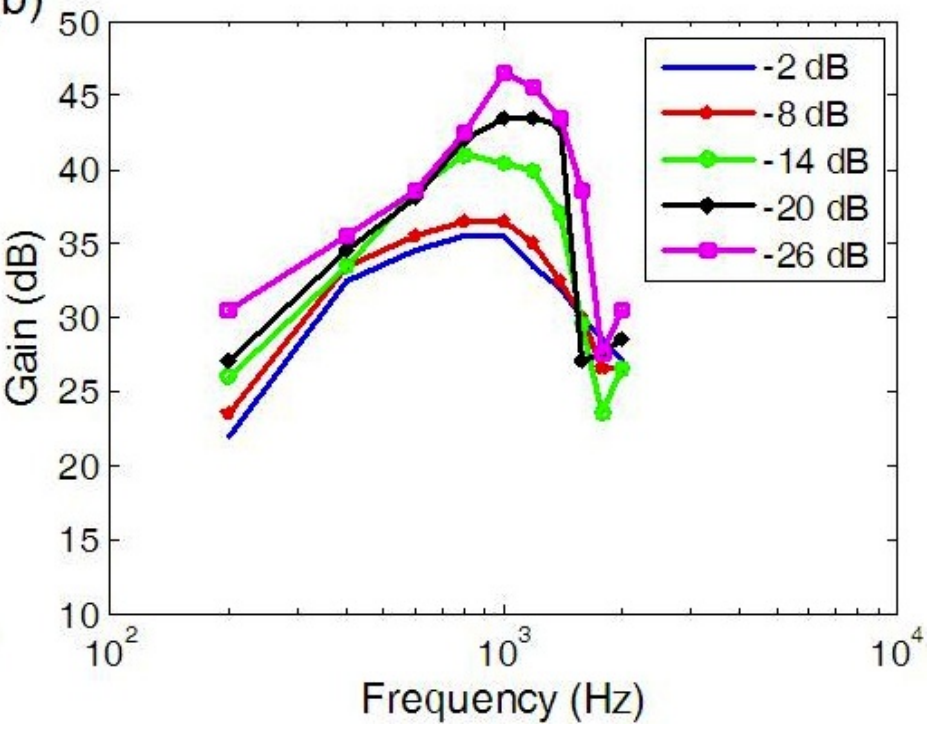

FiguRE 6: Frequency response (a) and the gain (b) of a single resonator at a particular place in the cochlea model. 
see the characteristic steep slope after resonance as seen in Figure 2. We also see the shift in resonant frequency from low to high as the input signal intensity decreases. Hardware limitations prevented us from covering the same dynamic range as shown in Figure 2; however, we showed that the model produces similar responses to the measured cochlea $[4,5]$.

\section{Conclusions}

We have presented a mathematical model of the cochlea which exhibits the same dynamical behaviour as the measured cochlea. The model was devised through qualitative analysis of the results of the measured cochlea. Mathematical analysis of the model shows that it possesses the dynamical properties of a Hopf bifurcation. This model has subsequently been used to gain greater insight into the mechanisms of hearing.

Acknowledgements This work was supported by ARC Discovery Project 0986025 .

\section{References}

[1] S. Camalet, T. Duke, F. Julicher, and J. Prost, Auditory Sensitivity Provided by Self-Tuned Critical Oscillations of Hair Cells. Proceedings of the National Academy of Sciences of the United States of America 97, 2000, pp. 3183-3188. C301, C302

[2] V. M. Eguíluz, M. Ospeck, Y. Choe, A. J. Hudspeth, and M. O. Magnasco, Essential Nonlinearities in Hearing. Physical Review Letters 84, 2000, 5232-5235. C301, C302, C305 
[3] E. Fragniere, Analogue VLSI Emulation of the Cochlea, Doctoral Thesis, Département d'électricité, EPFL, Lausanne, 1998. http://library.epfl.ch/en/theses/?nr=1796 C306

[4] T. J. Hamilton, C. Jin, A. van Schaik, and J. Tapson, An Active 2-D Silicon Cochlea. Biomedical Circuits and Systems, IEEE Transactions on 2, 2008, 30-43. C310, C313

[5] T. J. Hamilton, J. Tapson, C. Jin, and A. van Schaik, Analogue VLSI implementations of two dimensional, nonlinear, active cochlea models, Biomedical Circuits and Systems Conference, 2008. IEEE, 2008, pp. 153-156. C310, C313

[6] A. Kern, J.-J. van der Vyver, and R. Stoop, Towards a biomorphic silicon Hopf cochlea, Proceedings of the 10th IEEE conference on nonlinear dynamics of electronic systems, 2, 2002. C301, C302

[7] A. Kern, and R. Stoop, Essential Role of Couplings between Hearing Nonlinearities, Physical Review Letters 91 (12) 2003, 128101. C301, C302

[8] M. O. Magnasco, A Wave Traveling over a Hopf Instability Shapes the Cochlear Tuning Curve, Physical Review Letters 90 (5) 2003, 058101. C302

[9] C. J. Plack, The Sense of Hearing, Lawrence Erlbaum Associates, Inc., New Jersey, 2005. C301

[10] M. A. Ruggero, Responses to sound of the basilar membrane of the mammalian cochlea. Current Opinion in Neurobiology 2, 1992, 449-456. C304

[11] S. H. Strogatz, Nonlinear Dynamics and Chaos, Westview Press, Cambridge, 1994. C310

[12] J. Tapson, T. J. Hamilton, C. Jin, and A. van Schaik, Self-Tuned Regenerative Amplification and the Hopf Bifurcation, IEEE 
International Symposium on Circuits and Systems (ISCAS), Seattle, USA, 2008. C309

[13] A. van Schaik, and E. Fragniere, Pseudo-voltage domain implementation of a 2-dimensional silicon cochlea, International Symposium on Circuits and Systems (ISCAS), Sydney, Australia, 2001, pp. 185-188 vol. 2 doi:10.1109/ISCAS.2001921277 C306

\section{Author addresses}

1. T. J. Hamilton, School of Elec. Eng. \& Telecoms, University of NSW, Sydney, Australia.

mailto:t.hamilton@unsw. edu.au

2. J. Tapson, University of Cape Town, South AfricA. mailto: jonathan.tapson@uct.ac.za

3. M. Rapson, University of Cape Town, South AfricA. mailto:michael.rapson@uct.ac.za

4. C. Jin, University of Sydney, Australia. mailto:craig@ee.usyd.edu.au

5. A. van Schaik, University of Sydney, Australia. mailto: andre@ee.usyd.edu.au 